\title{
FISIOLOGÍA Y PERSPECTIVISMO EN LA CONCEPCIÓN LEIBNIZIANA DE LA SUSTANCIA CORPÓREA ${ }^{1}$
}

\author{
Miguel Escribano Cabeza \\ Universidad de Granada
}

\begin{abstract}
RESUMEN: Este trabajo pretende analizar la cuestión del perspectivismo leibniziano en clave biológica. Siguiendo esta idea, la actividad de la sustancia corpórea se interpretaría a partir de la relación individuo-entorno. En estos términos, el análisis de la operatividad del organismo nos lleva a la caracterización de lo que denominaremos vitalismo transcendental. A cada ser vivo, en función de su pertenencia a una u otra especie biológica, le corresponderá entonces un peculiar esquematismo a partir del cual el individuo comprende el entorno físico como mundo de percepción. Una ventaja de esta interpretación es aportar una mayor comprensión a la idea de la armonía preestablecida. Nos centraremos fundamentalmente en textos de madurez del filósofo.
\end{abstract}

PALABRAS CLAVE: Perspectivismo; máquina natural; vitalismo; mundo de percepción; círculo vital.

\begin{abstract}
The present work aims to analyze Leibniz' perspectivism in a biological note. Following this idea, the activity of the bodily substance will be interpreted as based on the relationship between the individual and the environment. Against this background, the analysis of the organism's functionality leads us to a characterisation which we have dubbed transcendental vitalism. Each living organism belonging to any biological species comprehends the physical environment as a world-of-perception. One of the advantages of such an interpretation is that it contributes to a better understanding of the idea of the pre-established harmony. To this end, we will focus primarily on those texts of the mature Leibniz.
\end{abstract}

KEYWORDS: Perspectivism; natural machine; vitalism; the-world-of-perception; vital circle.

\section{El orden de los fenómenos naturales y sus condiciones}

En la correspondencia que Leibniz mantuvo con Bernouilli y De Volder nos encontramos con un malentendido que resulta esclarecedor a la hora de entender la teoría leibniziana de las sustancias corporales. Tanto Bernouilli como de Volder criticaron a Leibniz esa necesidad suya de introducir sustancias

1 Este trabajo se ha realizado en el marco del proyecto "Leibniz en español" (FFI2014-52089-P). 
en la naturaleza, pues defendían la suficiencia de las fuerzas derivativas para explicar cualquier fenómeno natural, incluido el de la unidad de los cuerpos complejos. ${ }^{2}$ Leibniz está de acuerdo con Bernouilli y De Volder en que a cierto nivel es suficiente con la consideración físico-mecánica de los fenómenos corporales. Sin embargo, el problema hacia el que señala Leibniz, en el que sus corresponsales no acaban de entrar, no es el de la explicación científica del orden que observamos en la naturaleza sino el de las condiciones de posibilidad de tal orden. ${ }^{3}$ Ni Bernoulli ni De Volder entendieron la aplicación que hace Leibniz de la noción de forma al mundo físico y cómo a través de ella podemos llegar a distinguir lo que sean o no sustancias. ¿Cómo entonces iban comprender que son precisamente estas sustancias el origen del orden y la armonía que observamos en el mundo? Sirvan los siguientes fragmentos para ilustrar la controversia y los malentendidos a los que dio lugar.

[Esta es la respuesta que da Leibniz a una primera duda de Bernouilli] “¿es el principio activo la extensión o un modo de la extensión o es, más bien, una substancia distinta de la extensión?” Respondo que a mí me parece que es algo anterior a la extensión, algo constitutivo de la substancia misma que está en lo extenso. Es decir, la extensión no es, para mí, más que un atributo del agregado que resulta de muchas substancias. Por lo tanto, el principio de actividad no puede ser la extensión, ni un modo de ésta, ni actúa sobre la extensión sino en lo extenso. ${ }^{4}$

[Dice Bernouilli a Leibniz] Respondo ahora brevemente a lo demás de tu carta. Los cartesianos concederán que en el interior del sílice y demás cuerpos inanimados pueden contenerse otros animálculos, como los gusanos en el queso; pero, lo mismo que los gusanos no componen la materia del queso, o sea, lo que propiamente es el queso puesto que o han

${ }^{2}$ AA III 7, p. 860; OFC 16A, p.472.

3Para la posición de Leibniz: GP II, p. 250-254; OFC 16B 1198-1202.

${ }^{4} \mathrm{GP}$ II, p. 187; OFC 16B, p. 1114-1115. 
sido introducidos desde fuera o generados de óvulos o semillas, así también negarán que aquellos animálculos, que quizás se hospedan en el sílice, hagan por ello la materia del sílice, o sea, lo que propiamente es el sílice. ${ }^{5}$

[... y contesta Leibniz a De Volder] No pueden serme sus cartas poco gratas pues siempre me enseñan algo o me ofrecen la ocasión de profundizar o aclarar mis explicaciones. Dice Vd. «haber encontrado en la última mía muchas cosas inesperadas». Pero tal vez descubra Vd. que ya habían sido suficientemente insinuadas en otras anteriores, y sólo los prejuicios le impidieron llegar a las mismas conclusiones y no buscar ya la substancia y la fuente de las fuerzas allí donde no está. Por eso me he visto obligado a insistir más expresamente y responder, si no a lo preguntado, a lo que debía preguntarse. $^{6}$

\section{La complejidad de la materia y la distinción entre los cuerpos}

Ya en sus primeras cartas a Thomasius el joven Leibniz expuso uno de los objetivos centrales que marcan su proyecto de construir una Nueva Física: se trata de compaginar la noción mecanicista de figura con las formas aristotélicas. ${ }^{7}$ Leibniz concibe el cuerpo como un sistema corpuscular donde la forma es la función que integra el conjunto de las tendencias de las partes componentes, garantizando la unidad de su complejidad y la coherencia de su dinámica. La forma es un principio estructural, activo e intrínseco, que se identifica con la potencia de la materia, y que explica la educción en ella de una serie de estados estructurales, la unidad de esta serie y el paso de un estado a otro. Para escapar a la teoría de la potencia aristotélica y al mismo tiempo a la concepción cartesiana de la fuerza, la estrategia de Leibniz consiste en combinar, incluso en identificar, potencia o fuerza y forma, síntesis para la cual los 
pensadores ingleses desde Bacon a Hooke habían utilizado términos como textura, esquematismo o energía. ${ }^{8}$

Por tanto, y contestando las dudas planteadas por Bernouilli y De Volder, los cuerpos están en posesión de una complejidad y una dinámica internas, una formalidad que da cuenta de la articulación en ellos de las fuerzas derivativas que los organiza y a partir de la cual podemos explicar el conjunto de los fenómenos en sus diversas manifestaciones (fenómenos físicos, químicos, fisiológicos y biológicos).

A partir de aquí Leibniz distingue entre los siguientes niveles de complejidad corporal:

(a) los agregados: reunión de individuos que no constituyen una complexión o entidad al carecer de un principio formal que les integre y confiera una verdadera unidad (ej. un rebaño, un ejército).

(b) las complexiones o entidades: combinaciones cuyas partes se encuentran integradas bajo una forma común pero su constitución y su actividad dependen de un agente exterior; pueden ser naturales (ej. cristales, gemas, nieve, nitro, vitriolo) o artificiales, es decir, productos de algún ser vivo (ej. diques de los castores, celdas de las abejas).

(c) las máquinas naturales: agregados de cuerpos (complexiones naturales y artificiales) que posibilitan entornos de relación entre individuos de la misma o de distinta especie (ej. estaques, jardines, ciudades, pero también,

${ }^{8}$ Escribano (2016), cap. Il.1. 
veremos, los sílices y quesos de Bernoulli).

(d) organismos vivos: entidades que poseen un principio interno de organización y regulación de su complexión o de su formalidad según directrices finales; son estos cuerpos de los que se dice propiamente que son entidades o sustancias.

A partir de aquí, la idea de este trabajo es intentar reconstruir la imagen del mundo natural que subyace en la consideración que Leibniz hace a Bernoulli y De Volder con respecto a la relación entre el queso y sus gusanos o el sílice y sus animáculos. Nos serviremos para ello de una serie de equivalencias entre nociones leibnizianas y nociones pertenecientes a la biología, en concreto al pensamiento de J. von Uexküll.

\section{Seres vivos y entornos de agregación. Diferencia entre entorno}

\section{físico y mundo de percepción}

Un buen texto que da prueba de las investigaciones leibnizianas sobre la dinámica y la complexión de los entornos de agregación de las entidades (sustanciales o no) es la Protogaea. Al comienzo del texto Leibniz expone uno de los principales objetivos del estudio que tiene previsto llevar a cabo: se trata de sacar a la luz esa conformidad que aúna a individuos bajo una misma especie, cómo, mostrando las dependencias que tales individuos guardan con su medio entorno (y viceversa: mostrar las relaciones del entorno físico con las diversas especies biológicas y con las poblaciones de individuos que las pueblan o poblaron). Encontramos de este modo una doble relación de dependencia, una 
armonía, entre los procesos de organización del entorno físico y los que permiten a las especies que lo habitan o habitaron cumplir sus funciones vitales, a pesar de cada uno de ellos obedecer a causalidades diferentes: la corteza terrestre remite al núcleo volcánico del planeta y al movimiento violento de las aguas (que "trabajan como el laboratorio del químico"), las especies que habitan esa corteza a relaciones funcionales interespecíficas. Veamos algunas citas de esta obra que ilustran esta relación entre los seres vivos (individuos, especies o poblaciones) y el entorno físico-geográfico.

[...] la obra de la sal unida a la acción del calor reduce a limo las rocas duras para provecho de plantas y animales. ${ }^{9}$

[en el contexto del estudio de los fósiles, de su origen y su naturaleza, que Leibniz no duda provenir de animales antaño vivos, dice el filósofo lo siguiente] Pero ¿quién ha explorado esos apartados y ocultos abismos subterráneos? ¿Cuántos nuevos animales antes desconocidos nos ofrece el Nuevo Mundo? También parece verosímil que, con cambios tan importantes [cambios geológicos en la corteza terrestre], el aspecto de muchos animales se haya modificado. ${ }^{10}$

Creemos que, o bien esos animales [se refiere a los elefantes y argumenta en relación a los fósiles que se han localizado] estaban en la antigüedad más ampliamente extendidos por el mundo de lo que lo están hoy en día y que su naturaleza o la del suelo ha cambiado, o bien hay que pensar que fueron arrebatados a su lejanísima patria por el ímpetu de las aguas. ${ }^{11}$

[paralelo en la historia del medio físico-geográfico y de las poblaciones que lo habitan o habitaron] Debemos ocuparnos ahora de otros vestigios de grandes mutaciones de la naturaleza seguramente anteriores a la presencia del hombre tanto en nuestro país como en otros países. En opinión de Aristóteles y de Peiresc, Egipto se debe al Nilo, la campiña de Arles se debe al Ródano y Nannius sostiene que Holanda es un regalo de las tempestades del Rhin. Pues, efectivamente, los ríos despojan a las tierras superiores de la

9 Dutens II 2 203; OFC 8211. 
materia que transportan y Frisia se acrecienta cada día con nuestras pérdidas. $^{12}$

[mediación de la técnica en la relación individuo - medio entorno] Actualmente la técnica ha hecho tales progresos que en algunos sitios se ven tierras tomadas al mar, prados por debajo de los ríos como si el agua estuviera suspendida en aire y el campo protegido por un dique larguísimo de los desbordamientos de los ríos. Tal es el empeño de los mortales que la faz del mundo ha sido cambiada y creo que una gran parte de la tierra que habitamos se debe al propio esfuerzo de los hombres. Si bien hay que reconocer que también los castores, animales industriosos, dominan la técnica de construir diques y estanques. ${ }^{13}$

Leibniz reconoce que el agregado de materia que es nuestro planeta se encuentra en posesión de una estructura y orden, constituye un sistema cerrado en continua transformación, bien por su propia actividad, bien por los seres vivos que lo pueblan. Ahora bien, no es esta formalidad presente en la materia inerte ni por asomo similar a la de los cuerpos orgánicos, y ambas deben distinguirse con claridad. ${ }^{14}$ Así, llegados a este punto, nos dice Leibniz en la Protogaea que "hay que considerar si puede decirse que el animal es parte de la materia, como el pez es parte de la piscina, o el buey es parte de la manada". ${ }^{15}$ Es exactamente aquí donde nos detuvimos en la controversia con Bernoulli y De Volder.

\section{Esquematismo y círculo funcional o vital}

A diferencia del resto de complexiones corpusculares, las verdaderas

\footnotetext{
12Dutens II 2 233; OFC 8243.

${ }^{13}$ Dutens II 2, p. 234-235; OFC 8, p. 244.

${ }^{14}$ Dutens II, p. 221-222;OFC 8, p. 230-231.

${ }^{15} \mathrm{AA} \mathrm{VI} \mathrm{4,} \mathrm{p.} \mathrm{1671;} \mathrm{OFC} \mathrm{2,} \mathrm{p.} 222$.
} 
entidades, esto es, los seres vivos, se encuentran en posesión de un principio interno de actividad: su forma sustancial, que liga sus partes estructural y funcionalmente conforma a fines. Frente al mecanicismo, lo que en los seres vivos se enlaza en una unidad no es la figura sino la función de los distintos órganos. Este principio confiere a los seres vivos la autonomía que los caracteriza y les dota con la capacidad no sólo de reproducirse a sí mismo, sino además de regular y organizar su complexión interna con el objeto de mantenerse por sí mismos en la existencia.

Las plantas y animales o, por decirlo en una palabra, los cuerpos orgánicos que produce la naturaleza son máquinas aptas para perpetuar ciertas funciones, cosa que logran mediante la alimentación individual, en primer lugar, mediante la propagación de la especie, en segundo lugar, así como, por último, mediante su propia perfección, que cada cual lleva a cabo por medio de funciones específicas. ${ }^{16}$

La unidad fisiológica que permite pensar la singular complexión que caracteriza a los seres vivos y que introduce en la naturaleza ese ingrediente que es la percepción es el órgano. El órgano es el elemento mínimo estructural de los cuerpos vivos cuya función es reaccionar a, distinguir, integrar un conjunto de caracteres del entorno físico-geográfico.

Vemos también que la naturaleza ha dado percepciones destacadas a los animales, por el cuidado que ha puesto en dotarlos de órganos que reúnen varios rayos de luz o muchas ondulaciones del aire, para que por esa reunión sean más eficaces. Algo similar ocurre con el olor, con el gusto y con el tacto, y quizá también con otros muchos sentidos que nos son desconocidos. Luego explicaré cómo lo que sucede en el alma representa lo que ocurre en los órganos. ${ }^{17}$

${ }^{16}$ Dutens II, p. 171; OFC 8, p. 311.

${ }^{17} \mathrm{GP} \mathrm{VI}$, p. 611; OFC 2, p. 331. 
Estos caracteres se denominan por ello estímulos; por supuesto que el entorno físico presenta muchos otros caracteres que son para el órgano indiferentes. No sólo distintos órganos de un mismo cuerpo vivo reaccionan a conjuntos de caracteres diferentes de un mismo entorno, sino que también las distintas especies biológicas reaccionan a un conjunto de estímulos diferentes, aunque puedan presentarse todos ellos dentro del mismo entorno. El modo como el órgano reacciona a un estímulo, estableciendo una serie de correspondencias formales entre el conjunto de caracteres y su propia complexión, da lugar a la integración de tales caracteres en lo que denominamos una impresión sensible. Si bien las impresiones sensibles son efecto de la formalidad dinámica del órgano, son al mismo tiempo materia sobre la que opera la memoria. La memoria no reside en un órgano "superior", es antes bien la facultad presente en los organismos más complejos que es capaz de, y nace de, la correlación de los órganos sensibles (pasivos o receptores) y los órganos motores (activos o efectores): las correspondencias que en una percepción observamos entre impresiones sensibles procedentes de órganos distintos tiene lugar con ocasión de los hábitos que desinhiben cuando estimulan nuestra memoria, abriendo sobre el estado de percepción presente un abanico de disposiciones futuras que se concretará cuando se lleva a cabo la acción.

Veamos una serie de citas relativas a la naturaleza de la memoria y a la relación entre órganos sensoriales y efectores. 
relieve y distinción en las impresiones que reciben y, por consiguiente, en las percepciones que las representan $[\ldots]$ puede llegarse hasta el sentimiento, es decir, hasta una percepción acompañada de memoria; o sea, una percepción de la que durante largo tiempo perdura un cierto eco para dejarse oír ocasionalmente; y a ese viviente se le llama animal, así como a su mónada se le llama alma. ${ }^{18}$

[...] la vida o Entelequia primera es algo más que cualquier simple conato muerto, pues pienso que en ella, como en un animal, se alberga la percepción y el apetito, que responden ambas al estado presente de los órganos. ${ }^{19}$

Esta representación está enlazada con una reacción, o sea, un conato o apetito, a tenor de este conocimiento de su obrar. ${ }^{20}$

La memoria proporciona a las almas una especie de consecución, que imita a la razón, pero de la cual debe distinguirse. Por eso vemos que los animales, cuando perciben un objeto que les hiere y del que han tenido anteriormente una percepción similar, esperan, por la representación de su memoria, lo que iba unido a ese objeto en la percepción anterior y se ven impelidos a tener sentimientos similares a los que entonces tuvieron. Por ejemplo, cuando se muestra un palo a los perros, se acuerdan del dolor que les ha causado, y entonces aúllan y huyen (Discurso Preliminar, \65). 27. Y la fuerte imaginación que los impresiona y conmueve proviene de la magnitud o de la multitud de las percepciones precedentes. Pues muchas veces una fuerte impresión produce de golpe el efecto de un hábito prolongado o de muchas percepciones medianas reiteradas. 28. Los hombres actúan como los animales en la medida en que las consecuciones de sus percepciones sólo se realizan por medio del principio de la memoria; se parecen a los médicos empíricos, que poseen una simple práctica sin teoría; y nosotros somos sólo empíricos en las tres cuartas partes de nuestras acciones. ${ }^{21}$

Afirma además Leibniz que "no hay memoria sin caracteres o imágenes". ${ }^{22}$ Estos caracteres, señales o símbolos juegan un papel esencial para los seres vivos, ya que a través de ellos su cuerpo orgánico comprende e

${ }_{18} \mathrm{GP} \mathrm{VI,} \mathrm{p.} \mathrm{599;} \mathrm{OFC} \mathrm{2,} \mathrm{p.} 345$.

${ }^{19} \mathrm{AA}$ III 7, p. 963; OFC 16B, p. 514.

${ }^{20} \mathrm{AA}$ VI 4, p. 1507-1508; OFC 2, p. 143-144.

${ }^{21} \mathrm{GP}$ VI, p. 611; OFC 2, p. 365.

${ }^{22} \mathrm{AA}$ VI 3, p. 514; OFC 2, p. 113. 
interpreta el medio entorno, que deviene así mundo de percepción. ${ }^{23}$ A través de estos símbolos la memoria correlaciona un conjunto de impresiones sensibles con su complemento activo; la memoria por tanto tiene una actividad simbólica, al correlacionar señales y operaciones. Es así que el mundo de percepción del animal tiene un carácter semiológico: percepción y comportamiento se encuentran en él correlacionados formando un círculo que va desde el entorno físico, portador de características, hasta la memoria a través del órgano sensorial y vuelve desde la memoria a través de los órganos efectores hasta los objetos del entorno portadores de características. Por tanto, repitiendo lo dicho una vez más, en el mundo de percepción se encuentran ligados comportamiento y percepción: "en los cuerpos animados”, dice Leibniz, "lo orgánico responde a lo vital $\{y\}$ el movimiento responde a los apetitos". ${ }^{24}$

Sabemos, y así nos lo ha mostrado Leibniz, que el funcionamiento de este círculo, que podemos denominar círculo vital o funcional (siguiendo la terminología de J. von Uexküll), ${ }^{25}$ integra el conjunto de sus esquematismos percepción-operación en torno al cumplimiento de una serie de fines que coordinan las funciones desplegadas por cada uno de ellos. Esta correlación entre fines y funcionalidad orgánica es una variable de especie que Leibniz denomina forma sustancial. La forma sustancial, o fuerza activa primitiva, consiste en la ley que engloba o integra la serie de los estados vitales que correlacionan

${ }^{23}$ Tomamos el término mundo de percepción como equivalente leibniziano de Umwelt (UUXLÜLL 1920: Sechste Briefe). 
en un cuerpo series de estímulos y de operaciones. El animal, por pertenecer a tal especie, se encuentra en posesión de una forma sustancial a través de la cual comprende su entorno como mundo de percepción.

$[\ldots]$ en todas las especies orgánicas debe haber alguna cosa que responda al alma, y que los filósofos han llamado forma sustancial, que Aristóteles denomina entelequia primera $[\ldots]$ debemos siempre reconocer en general que es necesario que todo esté lleno de tales especies, que contienen en ellas un principio de la verdadera unidad que posee analogía con el alma y que está unida a algo que tiene manera de cuerpo organizado; de otro modo, no encontraríamos sustancias en la materia y los cuerpos no serían más que fenómenos y como sueños bien regulados. ${ }^{26}$

[...] el alma, la forma, la vida, la entelequia primera, o como gustes llamarla[...] la entelequia o actividad primitiva, alma o vida. ${ }^{27}$

En el mundo natural las relaciones (de dominio mutuo, esto es, de acción y pasión) que establecen vínculos (sustanciales o no) entre los seres vivos en el entorno obedecen, como no podría ser de otra manera, a criterios funcionales: fisiológicos y ecológicos. No sólo cada parte del organismo ocupa un lugar en éste según su función, más aún, cada viviente tiene en la extensión una posición que se determina por el conjunto de prestaciones y contraprestaciones que lo vinculan a otros vivientes de su entorno junto a los que constituye una máquina natural o, en términos modernos, un ecosistema.

\section{Memoria, hábito, voluntad}

Desde su proyecto De Summa Rerum Leibniz buscó diferentes vías para 
explicar en términos meramente fisiológicos cómo tiene lugar en el cuerpo esta coordinación orgánica. Al comienzo optó por la teoría de los espíritus animales. En carta a Arnauld fechada en 1686 Leibniz exponía de la siguiente manera la explicación del papel de intermediarios que tales espíritus animales juegan en este modelo:

\begin{abstract}
Nos preguntamos lo que determina a los espíritus [espíritus animales] a entrar en los nervios de cierta manera; yo respondo que es tanto la impresión de los objetos, como la disposición de los espíritus y nervios mismos, en virtud de las leyes ordinarias del movimiento. Pero por la concordancia general de las cosas, toda esta disposición ocurre sólo cuando hay al mismo tiempo en el alma esta voluntad a la que tenemos costumbre de atribuir la operación..$^{28}$
\end{abstract}

La teoría de los espíritus animales, presentes en el sistema nervioso, permite pensar la conexión entre los órganos sensoriales y los efectores. Se atribuiría así a la excitación de estos espíritus animales del sistema nervioso la función de mediar el acceso que tiene todo organismo a los estímulos del entorno. Esta teoría fue puesta entre paréntesis por el Leibniz maduro, quien confiaba en el avance de la mecánica (dentro de sus límites) para explicar tal fenómeno. Le dice a Bernoulli que él aboga por una concepción de la memoria que pueda explicar la dependencia entre órganos sensoriales y órganos motores sin mediación de tales espíritus animales.

Parece, por ejemplo, que en nuestros órganos algunas operaciones consisten en continuas vibraciones insensibles que, aun pareciéndonos a nosotros en reposo, son excitadas por otras contrarias. De manera que el problema nos conduce a la fuerza elástica. Yo sospecho que la memoria, por ejemplo, consiste en cierta conservación de vibraciones, de manera parecida a como

${ }^{28 F i n s t e r ~(1997), ~ p . ~ 183-184 ; ~ O F C, ~ p . ~} 1475$. 
una campana tañida sigue conservando y reverberando el sonido - - - - - - - - . Así que no parece que tengamos necesidad del fluido ese que designan con el nombre de espíritus animales, pues éstos pueden reducirse a la fuerza elástica que, aun no siendo necesaria en la práctica, no debería minusvalorarse para un conocimiento integral del conjunto de las cosas - - . . . . . . . . . . . . Adiós y cuídate. ${ }^{29}$

Seguramente estaría de acuerdo con Stenon en que los nervios actúan mediante una cierta excitación; pero, si yo no me engaño, la excitación misma no puede explicarse sino transmitida a través de algún fluido, como las sogas se contraen con el agua y los cabellos con el calor. [...] Así como los cabellos se crispan y contraen con el calor, de la misma manera creo que algo parecido ocurre en la materia nerviosa bajo el influjo de algún fluido más sutil. Esto no se opone a la doctrina de los espíritus animales, y no creo que Stenon los negase. ${ }^{30}$

Como se puede observar, Leibniz sospecha de la existencia de un proceso no consciente que tendría lugar en el mismo órgano, por ser elástico, y que funcionaría según el modelo de la mecánica vibracional, estableciendo correspondencias armónicas entre las diversas excitaciones que desencadenarían una respuesta refleja en el mismo órgano o en otro. ${ }^{31}$ La misma complexión del órgano sensorial parece que actúa como una caja de resonancias con la cual ciertas excitaciones del medio entrarían en correspondencia estimulando una respuesta. Tal respuesta podría prolongarse en el tiempo cuando la actividad estimulada por la excitación es conservada, transmitida y/o transformada por el cuerpo orgánico. En función de este proceso (de la complejidad de las correspondencias armónicas entre estímulos) la respuesta observada en los

${ }^{29 M S ~ I I I, ~ p . ~ 885 ; ~ O F C, ~ p . ~ 16 B, ~ p . ~ 941-942 . ~}$

30MS III, p. 864; OFC, p. 16B, p. 915, 929.

${ }^{31} \mathrm{El}$ exponente más importante de esta mecánica vibracional en la modernidad fue R. Hooke (ver: Escribano (2016) cap. II.1.) 
órganos motores va desde los actos reflejos o no conscientes a los conscientes que vinculamos con una voluntad.

Leibniz continuó en su madurez desarrollando estas reflexiones a partir de las nociones de memoria, hábito y voluntad. La correlación de los esquematismos senso-motores (percepción-comportamiento), que tiene su teatro de operaciones en la memoria, da lugar a lo que Leibniz denomina el hábito. El hábito, dice Leibniz, es la "consecución de las percepciones que se realiza por medio del principio de la memoria”, consecución a través de la cual tiene lugar la expresión de una voluntad. A partir de este principio de la memoria ${ }^{32}$ en el mundo de percepción del animal las impresiones sensibles se distinguen unas de otras, se organizan unas con relación a otras pero también, dice Leibniz, unas se hacen valedoras de una mayor fuerza que otras. Por tanto, que el mundo de percepción posee un carácter semiológico quiere decir que su comprensión implica una diferenciación paralela entre el ser vivo y su entorno, entre la adquisición de hábitos y la configuración de un hábitat. Estos esquemas que vinculan el mundo interior del animal y su entorno se presentan en las especies naturales conforme a una gradación que Leibniz expresa en términos de amplitud y riqueza de su mundo de percepción (que se corresponde con una gradación en perfección de los seres).

32GP VI, p. 611; OFC 2, p. 365. 


\section{El sistema de la Naturaleza}

Leibniz enlaza estas consideraciones relativas a la «dependencia metafísica» entre cuerpo y alma ${ }^{33}$ con lo que denomina «principio vital», siendo «vital» sinónimo de inmanente y de actividad primitiva. ${ }^{34}$

Admito efectivamente los principios de vida repartidos por toda la naturaleza, e inmortales, puesto que son substancias indivisibles o unidades, así como los cuerpos son multitudes destinadas a perecer por la disolución de sus partes. Estos principios de vida, o estas almas, tienen percepción y apetito. Cuando se me pregunta si son formas substanciales respondo distinguiendo: pues si este término se toma como lo toma el señor Descartes, cuando sostiene contra el señor Regis que el alma racional es la forma substancial del hombre, responderé que sí. Pero diré que no si alguien toma el término como los que se imaginan que hay una forma substancial de un trozo de piedra o de otro cuerpo no-orgánico; pues los principios de vida no pertenecen más que a los cuerpos orgánicos. Es verdad (según mi sistema) que no hay porción de la materia en la que no haya una infinidad de cuerpos orgánicos y animados; bajo los cuales incluyo no solamente a los animales y a las plantas, sino también quizá a otras clases que nos son enteramente desconocidas. Pero no hay que decir por ello que cada porción de la materia está animada, como no decimos que un estanque lleno de peces es un cuerpo animado, aunque el pez lo sea. Sin embargo, mi parecer sobre los principios de vida es en ciertos puntos diferente de lo que se ha enseñado acerca de ellos anteriormente. [...] Así, según mi sistema, las almas o los principios de vida no cambian nada en el curso ordinario de los cuerpos, y no dan tampoco a Dios ocasión de hacerlo. Las almas siguen sus leyes, que consisten en un cierto desenvolvimiento de las percepciones según los bienes y los males, y los cuerpos siguen también las suyas, que consisten en las reglas del movimiento; $y$, sin embargo, estos dos seres, de un género completamente diferente, se reencuentran juntos, y se responden como dos péndulos perfectamente bien reglados sobre el mismo pie, aunque quizá de una construcción muy diferente. Y esto es lo que yo llamo la armonía preestablecida, que descarta toda noción de milagro de las acciones puramente naturales, y hace que la marcha de las cosas esté reglada de una manera inteligible; mientras que el sistema común recurre a influencias absolutamente inexplicables. ${ }^{35}$

${ }^{33}$ Sobre esta idea: GP VI, p. 81, 621; GP III, p. 657-658; GP V, p. 162-163.

${ }^{34}$ Por ejemplo: GP VI, p. 396, 510, 512; OFC 8, p. 320, 292, 293.

${ }^{35} \mathrm{GP} \mathrm{VI,} \mathrm{p.} \mathrm{539-540;} \mathrm{OFC} \mathrm{8,} \mathrm{p.} \mathrm{324-325.}$ 
El vitalismo leibniziano (que habría de distinguirse de las posiciones animistas como las de Stahl o Hartsoeker que Leibniz critica) y su teoría de la sustancia corpórea, se funda en este acuerdo, armonía o correspondencia entre las afecciones del cuerpo y las percepciones del alma. ${ }^{36}$ En este trabajo hemos ensayado una interpretación de la dependencia o armonía cuerpo-alma a partir de la relación individuo-entorno. Esto, hemos visto, nos ofrece además la ventaja de facilitar la explicación de cómo en esa armonía preestablecida cuerpoalma ya está presente la idea de una armonía entre organismos de la misma y de distinta especie. Detengámonos ahora un poco más en ello.

No sólo existe una correlación armónica entre el animal y su mundo de percepción en conformidad con su complexión orgánica, sino que al mismo tiempo, y en relación con ésta, existe otra dependencia entre los individuos de una misma especie. Sólo así cada uno de ellos, y el conjunto como especie, pueden llegar a cumplir con la conformidad a fines que porta su ser orgánico (alimentarse, reproducirse...). Pero además, esta armonía se presenta entre especies distintas, algunos de cuyos círculos vitales se solapan en torno a determinados caracteres del entorno constituyendo cadenas funcionales donde estados vitales de unos organismos permiten la expresión de estados vitales de otros.

Lo que dije acerca de la conexión y simpatía de las substancias ha de aplicarse, creo, a todas ellas, aunque no sean de una misma naturaleza

${ }^{36}$ Sobre el vitalismo en Leibniz ver: Cardoso (2009) y (2012), y Nicolás (2011) y (2013). 
específica. ${ }^{37}$

No hemos explorado suficientemente cuál es el objetivo de la naturaleza en lo que respecta a los restantes cuerpos [...] cualquier cosa que pueda ser producida por las plantas y que resulte útil para el uso humano forma parte de sus fines, a los que tienden mediante determinados mecanismos; todo eso debe ser explicado antes que nada..$^{38}$

[...] los Reinos se comportan entre sí como fuente alimenticia, pero como por una escala, de manera que los minerales nutren a los vegetales, y éstos a los animales, y viceversa. Así que todo es medicina para todo ${ }^{39}$

[...] yo no desespero de que se pueda llegar a algo más profundo, si por lo que respecta a la teoría, por una parte, se continúa con las investigaciones de personas como Jungius, Malpighi, Hooke, Swammerdam y Leewenkoek y se les estudia con mayor atención, lo cual me asombra que no se haga, y por lo que respecta a la práctica médica, por otra parte, si cuando nuestro instinto y nuestras defensas naturales no bastan se recurre al instinto y a la ayuda de otros animales, pues en efecto, debido al modo artificial de vivir hemos perdido buena parte de nuestro instinto natural, que el médico se recetaba a sí mismo en el librillo de Galeno, a no ser que alguien quiera apelar además al consejo de los bárbaros, por estar más próximos a nuestra madre naturaleza. ${ }^{40}$

Leibniz busca una respuesta que logre explicar, por ejemplo, por qué el hombre bárbaro, como el animal, come instintivamente ciertas plantas para obtener ciertos beneficios de ellas, o cómo es que en el caso de la reproducción de las plantas un misma especie guarda relaciones de dependencia con otras para cumplir esta función (por ejemplo, con los insectos que las polinizan).

En las cosas que existen a la vez puede haber continuidad, aunque la imaginación no perciba más que saltos $[. .$.$] es necesario que todos los$ órdenes de seres naturales no formen más que una única cadena, en la que las diferentes clases, como otros tantos anillos, tienden tan estrechamente

37GP II, p. 233; OFC 16B, p. 1175.

${ }^{38}$ Dutens II, p. 171; OFC 8, p. 312, 311.

${ }^{39} \mathrm{AA} \mathrm{VI} 2$, p. 245, 246; OFC 8, p. 50 (la cita es de una obra de juventud, la Hyphotesis physica nova)

${ }^{40 D}$ Dutens II, p. 171; OFC 8, p. 312. 
unos a otros, que es imposible a los sentidos y a la imaginación fijar con precisión el punto en el que uno comienza o termina: todas las especies que bordean, o que ocupan por así decir regiones de inflexión o de retroceso, deben ser equívocas y estar dotadas de rasgos que pueden atribuirse igualmente a las especies vecinas. [...] no me extrañaría en absoluto averiguar que han sido encontrados seres, que con respecto a varias propiedades, por ejemplo, las de alimentarse, o multiplicarse, puedan pasar por vegetales con tanto derecho que por animales, y que conculcan las reglas habituales basadas sobre la suposición de una separación perfecta y absoluta de los diferentes órdenes de seres simultáneos que llenan el universo. ${ }^{41}$

No existe por tanto, así defiende Leibniz, una separación perfecta o absoluta entre las distintas especies ni entre los distintos reinos naturales, sino que sus círculos funcionales se encuentran solapados en torno a determinadas características del entorno físico respecto a las que el desempeño de esas funciones resulta convergente y establece entre una serie funcional y otra relaciones de correspondencia expresiva. Y es así que, por tanto, en un mismo entorno cohabitan una multiplicidad de individuos y especies interdependientes y que lo hacen ordenadamente, con armonía, como espejos vivientes que se reflejan unos a otros.

\section{Conclusiones}

Frente al mecanicismo, que reduce a relaciones de causa-efecto todos los fenómenos naturales, Leibniz defiende una biología experimental que analiza al mismo tiempo la naturaleza y los vivientes dentro de una lógica parte-todo; se trata de una perspectiva sistémica y funcional. La base del método la 
encontramos en el Arte Combinatoria. Se trata de establecer relaciones de correspondencias formales (respectos entre elementos) y sistémicas (parte-todo) entre distintas complexiones orgánicas según criterios funcionales y finales. En el caso de los seres vivos, hemos visto, estos criterios hace referencia a la conformidad, adaptación o ligazón que se tienden entre unas especies y otras y que permiten el cumplimiento de sus funciones vitales y a través de ello de su misma perfección. Así, decía Leibniz «cualquier cosa que pueda ser producida por las plantas y que resulte útil para el uso humano forma parte de sus fines, a los que tienden mediante determinados mecanismos», mecanismos que, dice más abajo, pasan desde una relación instintiva con la naturaleza (como el caso de los bárbaros) a los tratados de los médicos, donde se explicita estas relaciones y se procede a su estudio.

Concluyendo, ni Bernoulli ni De Volder entendieron cómo el sílice o el queso podían ser algo más allá de su mero estar a la mano del hombre (investigador de la estructura interna de la materia o productor de derivados lácteos) y llegar a formar parte del mundo de percepción de otro ser vivo. Para el ser vivo su mundo de percepción es su esfera espacio-temporal. El orden de los fenómenos guarda entonces una relación inmanente con el espacio-tiempo, que no es algo exterior a ellos sino que nace desde cada uno de los sujetos percipientes, esto es, de las infinitas sustancias cuya naturaleza consiste en ser representativas. Leibniz transita desde una fisiología sensorial con base al estudio del órgano (estética trascendental en la que se distinguen las especies en función de su complexión orgánica) a una reflexión holística de la naturaleza (dialéctica trascendental, donde 
se incorpora la idea de mundo biológico). La diferencia con Kant es que en el caso de Leibniz estamos hablando de un sujeto que se comprende como vida, cuya complejidad orgánica que integra una serie de esquemas dinámicos sensomotores es una variable de especie.

\section{Ediciones de los escritos de Leibniz}

LEIBNIZ, G.W. (1768), Opera Omnia, L. Dutens (ed.), 6 vols, Ginebra, 1768 (reimpr. Olms, Hildesheim, 1989) (citado como Dutens).

(1875), Die philosophische Schriften von Gottfried Wilhelm Leibniz.

Ed. C.I. Gerhardt (7 vols.), Berlin. (Reimp. Hildesheim: Georg Olms, 1978) (citado como GP).

(1903), Opuscules et fragments inédits (extraits des manuscrits de la

Bibliothèque Royale de Hanovre), ed. L. Couturat. Paris (reimp. Hildesheim: Georg Olms Verlag, 1966) (citado como OFI).

(1923-), Sämtliche Schriften und Briefe. Ed. Deutsche Akademie

der Wissenschaften. Darmstadt/Leipzig/Berlin: Akademie Verlag, 1923(citado como AA).

(1962), Die matematischen Schriften. Ed. C.I. Gerhardt (7 vols.).

Berlin (citado como MS).

(1997), Der Briefwechsel mit Antoine Arnauld, R. Finster (ed.),

Hamburg (citado como Finster). 
como OFC).

\section{REFERENCIAS BIBLIOGRÁFICAS}

CARDOSO, A. (2009), "Vida e vivência em Leibniz", O que nos faz. pensar, 26: $31-44$. (2012a), "Do dinâmico ao vital”, Ponencia: Acción Integrada

Luso-española "El surgimiento de la ciencia moderna en Europa: G.W. Leibniz", Lisboa 13-14 de enero de 2012.

ESCRIBANO CABEZA, M. (2016), Complejidad y dinámica en la filosofía natural y la metafísica de G.W. Leibniz, Tesis doctoral, director: Juan A. Nicolás Marín, Universidad de Granada.

NICOLÁS, J.A. (2011), “Dimensión vitalista de la ontología leibniziana”, en Nicolás, J. A. \& Toledo, S. (eds.), Leibnizy las ciencias empíricas, Comares, Granada: 71-93. (2013), "Leibniz: de la biología a la metafísica vitalista", en Arana, Jo. (ed.), Leibnizy las ciencias, Plaza y Valdés: 179-211.

VON UEXKÜLL, J. (1920), Biologische Briefe an eine Dame, Berlin: Paetel. 